\title{
Hydrophobic Interaction of the Water-Soluble ABA-Type Block Copolymer Composed of 2-Hydroxyethyl Methacrylate and Ethylene Oxide and Its Conformational Transition
}

\author{
Masahisa IKEMI, Nobuyuki ODAGIRI, and Isao SHINOHARA \\ Department of Polymer Chemistry, Waseda University, \\ 3-4-1, Ohkubo, Shinjuku-ku, Tokyo 160, Japan.
}

(Received March 19, 1980)

\begin{abstract}
The water-soluble ABA-type block copolymer composed of a hydrophobic poly(2-hydroxyethyl methacrylate) and a hydrophilic poly(ethylene oxide) was synthesized. The correlation between the structure of the block copolymer in dilute aqueous solution and its hydrophobic interaction was studied in comparison with random copolymers whose hydrophobic groups are statistically distributed along the chain. Fluorometric measurements using ANS as a probe for hydrophobic region were carried out on the aqueous solution of the block copolymer in the monomolecular state. The hydrophobic domain structure of the monomolecular block copolymer is discussed with respect to the change in the thermodynamic parameters for the binding process of ANS to the polymer chain. The binding ability of the block copolymer for an ANS molecule was found to be larger than that of corresponding random copolymers. This indicates that the mode of the arrangement of hydrophobic groups along the chain affects the hydrophobic interaction and that the block copolymer forms the large hydrophobic region which results from the aggregation of these hydrophobic blocks. It was observed that the temperature dependence of the binding constant of the block copolymer displayed a sharp transition within a narrow temperature range, indicating that the structural change takes place within a molecule. The thermodynamic parameters for both states of the block copolymer below and above the transition temperature were determined independently from the temperature dependence of the binding constant. This means that the hydrophobic domain of the block copolymer becomes larger in the lower temperature range than in the higher, presumably because of the segregation between the hydrophobic and the hydrophilic blocks.
\end{abstract}

KEY WORDS Water-Soluble Block Copolymer / Telomerization / 2Hydroxyethyl Methacrylate / Poly(ethylene oxide) / Hydrophobic Interaction / Fluorescent Probe / Hydrophobic Domain / Segregation /

Polymers consisting of both the hydrophilic and hydrophobic portions have characteristic properties, ${ }^{1}$ often referred to as the amphiphilic property. Only a few papers, however, have been published on the relation between the polymer structure and this property. The condition for the occurrence of either the hydrophilic or the hydrophobic functions still remains unclear and so it is desirable to study more extensively the influence of intramolecular interaction on the solution properties.

It has been reported that the hydrophobic interaction of a water-soluble amphiphilic polymer is influenced not only by the chemical structure and composition of the hydrophobic groups but also by the distribution of these groups along the chain. ${ }^{2,3}$ It may thus be expected that the mode of arrangement of hydrophobic groups in a polymer chain affects the occurrence of hydrophobicity in polymers.

The study on the conformation and intramolecular interaction of block copolymers consisting of an incompatible homopolymer pair in dilute solution has been the subject of extensive investigation. ${ }^{4}$ It is known that block copolymers adopt an intrachain phase-separated structure under adequate conditions. With regard to the water-soluble block copolymer, however, a study has been rarely performed on the relation between conformation and intramolecular interaction, especially hydrophobic interaction in aqueous solution.

In this paper, the water-soluble ABA-type block 
copolymer composed of a hydrophobic poly(2hydroxyethyl methacrylate) (PHEMA) and a hydrophilic poly(ethylene oxide) (PEO) was synthesized. Fluorometric measurements using a fluorescent probe for hydrophobicity were carried out on the aqueous solution of the block copolymer at various temperatures. A discussion is made of correlation between the structure of the block copolymer and its hydrophobic interaction.

\section{EXPERIMENTAL}

\section{Materials}

1-Vinyl-2-pyrrolidone (VPy), $\alpha, \alpha^{\prime}$-azobisisobutyronitrile (AIBN), 1-butanethiol (BuSH), N,Ndimethylformamide (DMF), methanol, hexane, and benzene were purified by conventional methods. HEMA was distilled under reduced pressure in a nitrogen atmosphere and the fraction of bp $87^{\circ} \mathrm{C}(5$ $\mathrm{mmHg}$ ) was used. Tolylene-2,4-diisocyanate (TDI) was distilled under a reduced pressure in a nitrogen atmosphere and the fraction of $\mathrm{bp} 86^{\circ} \mathrm{C}(2 \mathrm{mmHg})$ was used. 8-Anilino-1-naphthalene sulfonate (ANS), 2-aminoethanethiol (AESH), ethyl cellosolve, and chlorobenzene of reagent grade were used without further purification. Commercial grade of PEO was used following reprecipitation purification from hexane.

\section{HEMA-VPy Copolymerization}

The methanol solution containing the desired amounts of AIBN, HEMA, VPy, and BuSH was put into ampoules. Each ampoule was sealed under vacuum less than $10^{-5} \mathrm{mmHg}$ after degassing three times. The sealed ampoules were immersed in an oil bath at $60 \pm 0.1^{\circ} \mathrm{C}$ and shaken vigorously. After the proper reaction time elapsed, the ampoules were removed from the bath and immediately frozen in liquid nitrogen. They were warmed sufficiently so as to melt the contents which were then poured into diethyl ether to precipitate copolymers. These were filtered off, washed thoroughly with the precipitant and dried in vacuo.

\section{Synthesis of HEMA-EO ABA-Type Block Co- polymer}

HEMA-AESH Oligomerization. The DMF solution containing the desired amounts of AIBN, HEMA, and AESH to act as a chain-transfer reagent was put into an ampoule. The ampoule was sealed by conventional methods and immersed in an oil bath at $60.0 \pm 0.1^{\circ} \mathrm{C}$ for a given period of time. The contents were then poured into ether to precipitate the oligomer. The resulting oligomer was filtered off and dried in vacuo.

Addition of TDI to PEO. The addition of TDI to PEO was performed under the condition of the reactive group ratio $[\mathrm{NCO}] /[\mathrm{OH}]=2.0$ in chlorobenzene at $80^{\circ} \mathrm{C}$ for 48 hours.

Block Copolymerization. An amino-semitelechelic oligo-HEMA (OHEMA) dissolved in DMF was allowed to react with an isocyanato-telechelic $\mathrm{PEO}$ in chlorobenzene at $0^{\circ} \mathrm{C}$ under the condition that the reactive group ratio was $[\mathrm{NCO}] /\left[\mathrm{NH}_{2}\right]=1.0$. After the proper amount of time elapsed, the reaction mixture was precipitated from diethyl ether, filtered off, and dried in vacuo.

Purification of Block Copolymer. Purification of the block copolymer was carried out by fractional precipitation based on the difference in temperature dependence of solubility toward ethyl cellosolve among these prepolymers and the block copolymer. The final product was dissolved in methanol and then poured into diethyl ether to precipitate the block copolymer. The resulting block copolymer was filtered off and dried in vacuo.

\section{Analysis of Oligomers and Copolymers}

The polymer structures were confirmed by infrared spectroscopy (Hitachi EPI G-III). The number-average molecular weight $\left(M_{n}\right)$ of the OHEMA was determined by a vapor pressure osmometer (Hitachi VPO 117) in a methanol solvent. The molecular weight of PEO was determined from the viscosity equation. ${ }^{5} \mathrm{~A}$ viscometric measurement was carried out with a modified Ubbelohde viscometer in a thermostatically-controlled reservoir. The composition of copolymers was determined by elemental analysis (Yanagimoto MT-2). The amino-group concentration was determined by the titration of acetic acid solution of amino-semitelechelic OHEMA with perchloric acid-acetic acid solution using crystal violet as an indicator. The isocyanato-group concentration was determined by back titration with an $\mathrm{HCl}$-methanol solution using bromophenol blue as the indicator, after the reaction of a given concentration of dibutylamine-chlorobenzene solution with isocyanato-telechelic PEO for 24 hours at room temperature had occurred. The composition of the 
block copolymer was determined by hydroxyl group analysis developed by Pommerening et al. ${ }^{6}$ The pyridine solution of the sample was allowed to react with acetic anhydride for 7 hours at $80^{\circ} \mathrm{C}$, with $\left[\mathrm{Ac}_{2} \mathrm{O}\right] /[\mathrm{OH}]=5.23$ in order to acetylate the hydroxyl groups of the sample. A water-dioxane mixture was added to the sample solution to hydrolyze the acetic anhydride. The hydroxyl group concentration was determined by back titration of acetic acid with an $\mathrm{NaOH}$-methanol solution using cresol red-thymol blue mixture as a mixed indicator.

\section{Fluorometric Measurement}

Fluorometric measurements were carried out with a spectrofluorometer (Hitachi MPF-4). The ANS of the sample solution was excited by an incident light of $360 \mathrm{~nm}$. Water from a thermostated bath was circulated through the water jacket around the cell to control the temperature of the sample.

\section{RESULTS AND DISCUSSION}

\section{Synthesis of HEMA-EO ABA-Type Block Co- polymer}

Table I shows the results on the oligomerization of HEMA using AESH as the chain-transfer reagent. The HEMA-AESH oligomerization proceeded through the telomerization mechanism as reported in the previous paper. ${ }^{7}$ The molecular weight determined from VPO was nearly equal to the molecular weight calculated from the amino-group analysis assuming that one molecule possesses one amino group. Consequently, it was confirmed that an amino-semitelechelic OHEMA, i.e., the oligomer possessing one amino group at one side end, was prepared.

The results on the preparation of the isocyanatotelechelic PEO are also shown in Table I. The addition of TDI to hydroxyl-telechelic PEO was performed when the reactive group ratio $[\mathrm{NCO}] /[\mathrm{OH}]$ was equal to 2.0 in chlorobenzene at $80^{\circ} \mathrm{C}$. The reaction was found to be complete after 48 hours and was followed by isocyanato-group analysis. The molecular weight of the final product determined from VPO was approximately equal to the calculated molecular weight, assuming that there was the addition of two TDI molecules to both ends of one PEO molecules. From these facts, it was confirmed that approximate two isocyanato groups were introduced to both ends of a PEO molecule.

HEMA-EO ABA-type block copolymer was synthesized by the reaction of the aminosemitelechelic OHEMA with the isocyanatotelechelic PEO. The amino-semitelechelic OHEMA dissolved in DMF was allowed to react with the isocyanato-telechelic PEO in chlorobenzene at $0^{\circ} \mathrm{C}$ for 48 hours with the reactive group ratio $[\mathrm{NCO}] /\left[\mathrm{NH}_{2}\right]=1.0$. The reaction mixture was precipitated from diethyl ether to obtain an HEMA-EO ABA-type block copolymer.

The solubilities of the constituent blocks toward ethyl cellosolve were remarkably different from each other. PEO dissolves in ethyl cellosolve only at elevated temperatures. On the other hand, OHEMA freely dissolves in ethyl cellosolve even at low temperatures. Thus, the block copolymer consisting of these prepolymers exhibits intermediate solubility properties. Furthermore, in the ethyl cellosolve solution of both the block copolymer and the PEO, the temperature at which the precipitate is formed by lowering temperature remarkably differs from

Table I. Preparation of prepolymers for HEMA-EO ABA-type block copolymer

\begin{tabular}{l} 
Prepolymer \\
\cline { 2 - 3 } \\
\cline { 2 - 3 }
\end{tabular}


the temperature at which the precipitate redissolves by elevating the temperature; the former is lower than the latter. These two critical temperatures of the block copolymer were lower than those of PEO.

Purification of the block copolymer was carried out by fractional precipitation based on the difference in temperature dependence of solubility toward ethyl cellosolve among these prepolymers and the block copolymer. The reaction product dissolved in an ethyl cellosolve solution containing $15 \mathrm{vol} \%$ hexane at an elevated temperature. This solution was gradually cooled to $0^{\circ} \mathrm{C}$ to precipitate the block copolymer and PEO. When this solution was heated to $28^{\circ} \mathrm{C}$, only the block copolymer was redissolved and PEO remained undissolved. This solution was then centrifuged for $60 \mathrm{~min}$ at $15000 \mathrm{rpm}$ in a fixedangle rotor kept at $28^{\circ} \mathrm{C}$ to remove the PEO. The supernatant containing the block copolymer and OHEMA was again cooled to $0^{\circ} \mathrm{C}$ to precipitate only the block copolymer. This solution was centrifuged in a fixed-angle rotor kept at $0^{\circ} \mathrm{C}$ to remove OHEMA. The block copolymer obtained as precipitate was redissolved in a common solvent such as methanol and then precipitated from diethyl ether to isolate the block copolymer. These procedures were repeated twice to obtain the purified block copolymer.

The results on the analysis of the purified block copolymer are shown in Table II. The sample of the block copolymer was freely soluble in water. The composition of the block copolymer determined from the hydroxyl-group analysis was approximately equal to the calculated theoretical composition, the architecture of the ABA-type being assumed. In the infrared spectrum of the block

Table II. Preparation of HEMA-EO ABA-type block copolymer ${ }^{\mathrm{a}}$

\begin{tabular}{|c|c|c|c|}
\hline \multicolumn{2}{|c|}{ Block chain length $\left(M_{n}\right)$} & \multicolumn{2}{|c|}{ HEMA mole fraction } \\
\hline HEMA $^{\mathbf{b}}$ & $\mathrm{PEO}^{\mathrm{c}}$ & Found $^{d}$ & Calcd \\
\hline 2640 & 7110 & 0.193 & 0.201 \\
\hline \multicolumn{4}{|c|}{$\begin{array}{l}\text { a }\left[\mathrm{NH}_{2}\right] /[\mathrm{OH}]=1 \text { at } 0^{\circ} \mathrm{C} \text { for } 48 \mathrm{~h} \text {; end-group concn, } 0.02 \\
\text { moll } 1^{-1} \text {. } \\
\text { b From amino-group analysis. } \\
\text { c Precursor molecular weight determined by viscosity } \\
\text { measurement prior to addition of TDI. } \\
\text { d From hydroxyl-group analysis. }\end{array}$} \\
\hline
\end{tabular}

copolymer, the peaks assigned to hydroxyl and carbonyl groups of HEMA were observed in approximate $3500 \mathrm{~cm}^{-1}$ and $1740 \mathrm{~cm}^{-1}$, respectively. The peak assigned to ether bond of PEO was also observed in $1100 \mathrm{~cm}^{-1}$. Furthermore, the peak in $2270 \mathrm{~cm}^{-1}$ assigned to isocyanato group of isocyanato-telechelic PEO disappeared. This confirms that the HEMA-EO ABA-type block copolymer was synthesized.

\section{Thermodynamic Analysis of the Complex Formation} of the Fluorescent Probe with the Water-Soluble Polymers

It is well known that ANS, the fluorescent probe for hydrophobicity, predominantly binds to hydrophobic region of polymers, resulting in the enhancement of fluorescence intensity and a blue shift in the maximum wavelength of emission, depending on the hydrophobicity around the binding site. Thus, the binding constants for the complex formation of ANS with water-soluble polymers can be determined by the fluorometric measurements made on the aqueous solution of polymers in the presence of ANS.

The constants for the binding of a fluorophore (F) to a polymer (P) with $n$ independent binding sites were determined from the following model, ${ }^{8}$

$$
\mathrm{P}+\mathrm{F} \stackrel{K}{\rightleftharpoons} \mathrm{C}_{\mathrm{f}}
$$

where $K$ is a binding constant for the formation of the binary complex $\mathrm{C}_{\mathrm{f}}$. If only the fluorophorepolymer complex possesses significant fluorescence, the following relationship holds at a fixed total concentration of the fluorophore, $F_{0}$, and an arbitrary concentration of the polymer, $P_{0}$,

$$
I / I_{\max }=\psi C_{\mathrm{f}} / \psi F_{0}=C_{\mathrm{f}} / F_{0}
$$

In eq $2, I$ is the observed intensity of fluorescence, $I_{\max }$ is the intensity of fluorescence when the polymer concentration is made infinitely large, and $\psi$ is a proportionality constant relating the fluorescence intensity to the concentration of the dyepolymer complex. By applying the law of mass action, the following equation is derived under the condition of $n P_{0} \gg C_{\mathrm{f}}$.

$$
F_{0} / I=1 / \psi+1 /(\psi n K)\left(1 / P_{0}\right)
$$

Plots of $\left(F_{0} / I\right) v s$. $\left(1 / P_{0}\right)$ give $1 / \psi$ and $1 / \psi n K$ from the value of the intercept and the slope, respectively.

Typical plots are shown in Figure 1 for the 


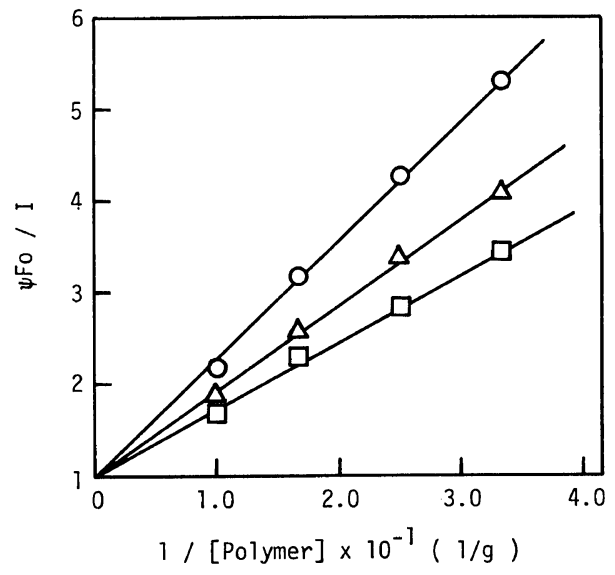

Figure 1. Typical plots of $\left(F_{0} / I\right)$ vs. $(1 / P)$ in HEMAVPy copolymer-ANS system: $O, 10^{\circ} \mathrm{C} ; \triangle, 20^{\circ} \mathrm{C} ; \square$, $30^{\circ} \mathrm{C}$; HEMA mole fraction in copolymer, 0.518 .

HEMA-VPy random copolymers (the detail description on the preparation of the HEMA-VPy random copolymers will be given in the following section). In this case, each plot was normalized by modifying eq 3 so that the values, extrapolated to 0 concentration, converged to unity on the ordinate. The plots display good linearity, showing that the binding course of ANS to a polymer chain seems to follow the Langmuir binding isotherm. Consequently, the first binding constant $n K$, often expressed as $K_{1}$, can be determined. From the $K_{1}$ value and its temperature dependence, the thermodynamic parameters for the complex formation can be obtained by eq 4,5 , and 6 .

$$
\begin{gathered}
\Delta G=-R T \ln K_{1} \\
\Delta H=-R \mathrm{~d}\left(\ln K_{1}\right) / \mathrm{d}(1 / T) \\
\Delta S=-\mathrm{d}(\Delta G) / \mathrm{d} T
\end{gathered}
$$

Hydrophobic Interaction of HEMA-VPy Random Copolymer

Table III shows the results of HEMA-VPy copolymerization using $\mathrm{BuSH}$ as the chain-transfer reagent. All the sample was freely soluble in water. PHEMA was insoluble and swelled only about $40 \%$ in water due to the hydrophobicity of the $\alpha$-methyl groups and the main chain. ${ }^{2}$ HEMA-VPy copolymers become soluble in water when the composition of a hydrophilic comonomer, VPy, exceeds $25 \mathrm{~mol} \%$.

Fluorometric measurements were carried out on the HEMA-VPy random copolymers in the presence of ANS. The first binding constant for the binding course of ANS to a polymer chain was determined from a graph of $\left(F_{0} / I\right)$ vs. $\left(1 / P_{0}\right)$ to obtain the free-energy change $(\Delta G)$ for the complex formation according to eq 4 . Figure 2 shows the temperature dependence of the $\Delta G$ value for the complex formation. The results show that the complex formation of ANS with copolymers becomes more stable with increasing temperature, indicating that the hydrophobic interaction plays an important role in the process of the complex formation. Figure 3 shows the relation between the $\Delta G$ value and the HEMA composition in the binding process of ANS to the copolymers at $20^{\circ} \mathrm{C}$. The $\Delta G$ value was found to decrease with increasing HEMA composition in the copolymers. Therefore, the complex of ANS with a polymer chain is considered to become more stable with an increase in the composition of hydrophobic HEMA units. Assuming that plots of the $\Delta G$ values in Figure 2 display a linearity against temperature within the temperature range in which measurements were carried out, the entropy change $(\Delta S)$ and the enthalpy change $(\Delta H)$ for the binding of ANS to a

\begin{tabular}{|c|c|c|c|c|}
\hline \multirow{2}{*}{$\begin{array}{l}\text { HEMA mole fraction } \\
\text { in monomer }\end{array}$} & \multirow{2}{*}[\mathbf{S}]{$/\left[\mathbf{M}_{1}\right]+\left[\mathbf{M}_{2}\right]$} & Time & Conversion & \multirow{2}{*}{$\begin{array}{l}\text { HEMA mole fraction } \\
\text { in copolymer }\end{array}$} \\
\hline & & $\min$ & $\%$ & \\
\hline 0.04 & 0.004 & 90 & 30.5 & 0.207 \\
\hline 0.08 & 0.004 & 100 & 26.4 & 0.346 \\
\hline 0.16 & 0.004 & 180 & 32.2 & 0.518 \\
\hline
\end{tabular}
polymer chain at $20^{\circ} \mathrm{C}$ were evaluated as shown in

Table III. Preparation of HEMA-VPy random copolymer ${ }^{\mathrm{a}}$

${ }^{a}[\mathrm{AIBN}]=5 \times 10^{-3} \mathrm{moll}^{-1}$ at $60^{\circ} \mathrm{C} ;\left[\right.$ Monomer] $=5 \mathrm{moll}^{-1}$ in $\mathrm{MeOH}$; [S], chain-transfer agent $(\mathrm{BuSH})$ concentration. 


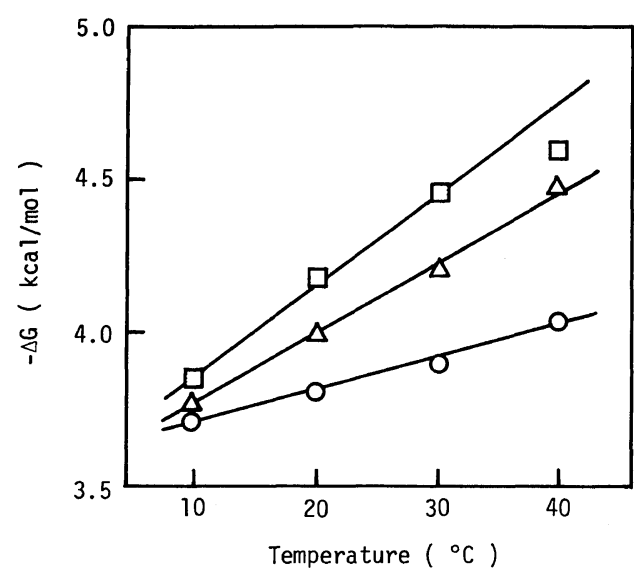

Figure 2. Temperature dependence of $\Delta G$ for the binding process of ANS to HEMA-VPy copolymers; HEMA mole fraction in copolymers: $\bigcirc, 0.207 ; \triangle, 0.346$; $\square, 0.518$.

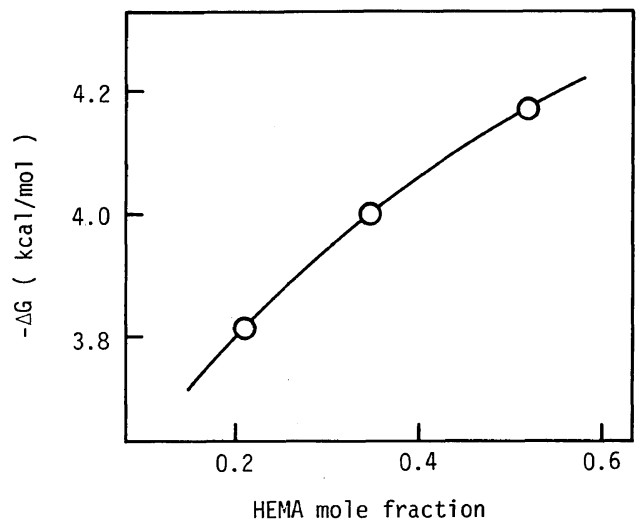

Figure 3. Relation between $\Delta G$ and HEMA composition in the binding process of ANS to HEMA-VPy copolymers at $20^{\circ} \mathrm{C}$.

Table IV. The values of $\Delta H$ and $\Delta S$ increased with increasing HEMA composition in the HEMA-VPy copolymers. This suggests that the iceberg around the hydrophobic groups increased with increasing the hydrophobic HEMA units and that the ordered structure of water around the binding site was remarkably destructed in the binding of ANS. Although the complex formation of ANS was unfavourable as for the enthalpy term, i.e., the $\Delta H$ value increases with the HEMA composition, the $\Delta G$ value decreased with increasing HEMA composition in copolymers, suggesting that the entropy term was a dominant factor in the binding of ANS
Table IV. Thermodynamic parameters for the binding process of ANS to HEMA-VPy random copolymer

\begin{tabular}{|c|c|c|c|}
\hline \multirow{2}{*}{ HEMA mole fraction } & $\Delta G^{\mathrm{a}}$ & $\Delta H$ & $\Delta S$ \\
\hline & cal & cal & e.u. \\
\hline 0.207 & -3815 & -656 & 10.8 \\
\hline 0.346 & -3998 & 2679 & 22.8 \\
\hline 0.518 & -4170 & 4559 & 29.7 \\
\hline
\end{tabular}

a At $20^{\circ} \mathrm{C}$.

to a polymer chain. These results indicate that the hydrophobic region becomes large with increasing HEMA composition in the HEMA-VPy copolymers, and is accompanied by an increase in the $\Delta H$ and $\Delta S$ values.

\section{Hydrophobic Interaction of the Block Copolymer and Its Intramolecular Conformational Change}

A fluorometric measurement was carried out on the aqueous solution of the HEMA-EO ABA-type block copolymer in the presence of ANS at various temperatures. The aqueous solution of the block copolymer possessed significant fluorescence in the presence of ANS. On the other hand, the aqueous solution of PEO, i.e., one of the constituent blocks, possessed no fluorescence of ANS even at a high polymer concentration, indicating that the PEO blocks had no binding affinity to ANS. Therefore, fluorescence from the block copolymer solution is considered to be attributable only to the ANS bound predominantly to the hydrophobic PHEMA block. Consequently, the fluorescence spectra of the block copolymer solution should reflect sensitively the micro-environment structure of the hydrophobic PHEMA domain and its structural change.

By the same procedure as described in the previous section, the first binding constant for the complex formation of ANS with the block copolymer was determined from the fluorescence spectra. The relation between the logarithm of the first binding constant, $\ln K_{1}$, and the reciprocal absolute temperature, $1 / T$, is shown in Figure 4. The drastic transition point was observed in the plots of $\ln K_{1}$ vs. $1 / T$ within a narrow temperature range between $15^{\circ} \mathrm{C}$ and $20^{\circ} \mathrm{C}$. This indicates that a remarkable change took place in the binding course of the fluorescent probe to the block copolymer within this 


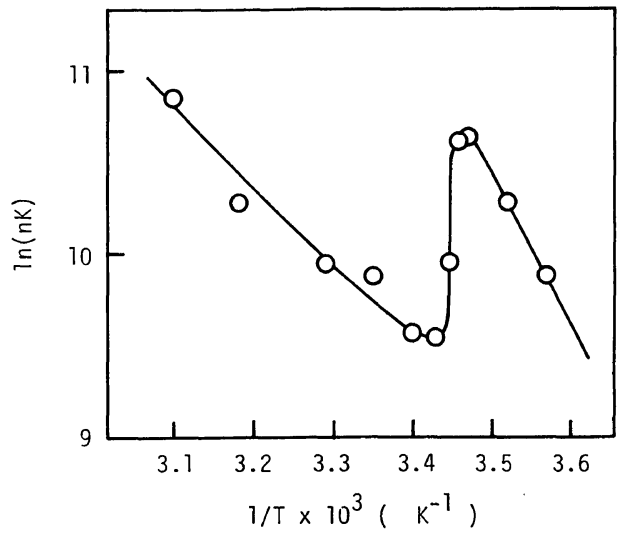

Figure 4. Relation between $\ln \left(K_{1}\right)$ and reciprocal absolute temperature in the binding process of ANS to the HEMA-EO ABA-type block copolymer; HEMA mole fraction, 0.193 .

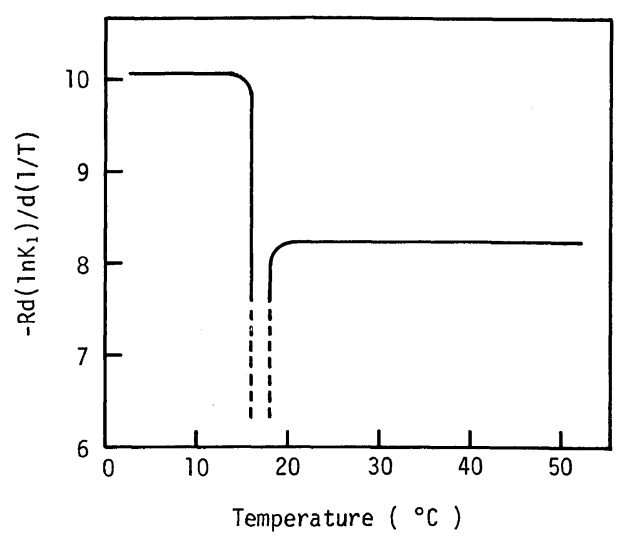

Figure 5. Relation between $-R \mathrm{~d}\left(\ln K_{1}\right) / \mathrm{d}(1 / T)$ and temperature in the binding process of ANS to the HEMA-EO ABA-type block copolymer; HEMA mole fraction, 0.193 .

temperature range. It should be noted that the block copolymer was in the monomolecular state within the range of the polymer concentration in which measurements were made, ${ }^{9}$ and that no transition of the thermodynamic parameters was observed in the random copolymer whose hydrophobic groups were statistically distributed along the chain. This drastic transition is considered to result from the intramolecular structural change in the hydrophobic blocks of the block copolymer.

As shown in Figure 5, the enthalpy change $(\Delta H)$ for the complex formation was estimated, assuming

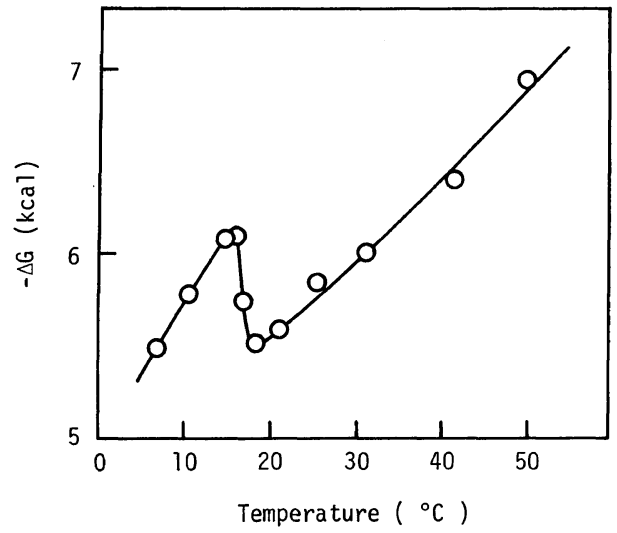

Figure 6. Temperature dependence of $\Delta G$ for the binding process of ANS to the HEMA-EO ABA-type block copolymer; HEMA mole fraction, 0.193.

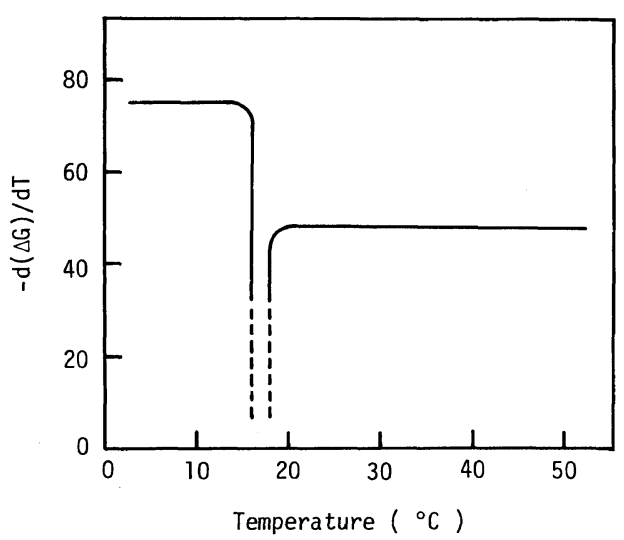

Figure 7. Relation between $-\mathrm{d}(\Delta G) / \mathrm{d} T$ and temperature in the binding process of ANS to the HEMA-EO ABA-type block copolymer; HEMA mole fraction, 0.193 .

that this curve displays a linear relationship against temperature before and after the transition. The values of $\Delta H$ were positive in both states, below and above the transition temperature, showing that the complex formation of ANS with the hydrophobic PHEMA block was energetically unfavourable. Moreover, the $\Delta H$ value of the lower temperature range was found to be larger than that of the higher temperature range. The $\Delta G$ values for the complex formation of ANS with the block copolymer were determined and the temperature dependence of the $\Delta G$ value is shown in Figure 6. The transition point 
was also observed in the same temperature range. The values of $\Delta G$ decreased with increasing temperature though the values of $\Delta H$ was positive, showing that the entropy term was positive in both states below and above the transition temperature. Consequently, it was revealed that the binding of ANS to the hydrophobic PHEMA domain is attributable to the hydrophobic interaction. Assuming that this curve displays a linear relationship against temperature before and after the transition, the entropy change $(\Delta S)$ was estimated as shown in Figure 7. It was found that the values of $\Delta S$ for the complex formation was considerably large and that the $\Delta S$ value of the low temperature range was larger than that of the higher temperature range. This means that the extreme destruction of the iceberg around the binding site took place in the binding process of ANS, especially within the lower temperature range.

The authors have investigated the structure of the polymolecular micelle of the block copolymer formed in the higher concentration range by means of small angle X-ray scattering. ${ }^{9}$ Consequently, it has been found that in the spherical micelle the large boundary region was formed in the interface between the hydrophobic core of the PHEMA blocks and the peripheral shell of the PEO blocks due to the partial mixing of the constituent blocks and that the structure of the boundary region was remarkably influenced by temperature. Therefore, the same event may exist even in the state of the monomolecular dispersion. The structure of the hydrophobic domain, if any, should be affected by the structural change of the boundary region, i.e., the degree of the segregation between the PHEMA and the PEO blocks.

The block copolymer possessed large values of both $\Delta H$ and $\Delta S$ for the binding of ANS to the hydrophobic PHEMA blocks compared with the corresponding random copolymers, indicating that this type of water-soluble block copolymer forms a large domain of the aggregated hydrophobic blocks even at relatively high temperatures. The fact that plots of $\Delta G v s . T$ and $\ln K_{1} v s .1 / T$ display a sharp transition state in which plots prominently deviate from a linearity shows that the hydrophobic domain structure of the block copolymer drastically changes within the critical temperature region. The increase in the values of $\Delta H$ and $\Delta S$ below the critical temperature suggest that the region with strong hydrophobicity was formed in the lower temperature range, presumably because of the segregation between the hydrophobic and the hydrophilic blocks.

\section{REFERENCES}

1. See, for example, (a) T. Okano, S. Nishiyama, I. Shinohara, T. Akaike, and Y. Sakurai, Polym. J., 10, 223 (1978); (b) M. Tollar, M. Stol, and K. Kliment, J. Biomed. Mater. Res., 3, 305 (1969); (c) F. J. Holly and M. F. Refojo, ibid., 9, 3061 (1975).

2. T. Okano, M. Ikemi, and I. Shinohara, Nippon Kagaku Kaishi, 93 (1977).

3. T. Okano, M. Ikemi, and I. Shinohara, Nippon Kagaku Kaishi, 1702 (1977).

4. See, for example, (a) T. Okano, M. Ikemi, and I. Shinohara, Polym. J., 10, 477 (1978); (b) M. Girolamo and J. R. Urwin, Eur. Polym. J., 7, 693 (1971); (c) H. Ohnuma, T. Kotaka, and H. Inagaki, Macromolecules, 7, 311 (1974); (d) A. Dondos, P. Rempp, and H. C. Benoit, Polymer, 13, 97 (1972); (e) idem., ibid., 16, 698 (1975).

5. S. Sadron and P. Rempp, J. Polym. Sci., 29, 127 (1958).

6. K. Pommerening, J. Stamberg, and P. Sedlacek, Collect. Czech. Chem. Commun., 33, 3191 (1968).

7. T. Okano, M. Katayama, and I. Shinohara, J. Appl. Polym. Sci., 22, 369 (1978).

8. J. L. Wang and G. M. Edelman, J. Biol. Chem., 246, 1185 (1971).

9. M. Ikemi, N. Odagiri, S. Tanaka, I. Shinohara, and A. Chiba, Macromolecules, 13(6), (1980), in press. 